\title{
A INGESTÃO DE PRÉBIOTICOS PREVINE A MALABSORÇÃO DE FERRO E ANEMIA INDUZIDAS PELA GASTRECTOMIA? ESTUDO EXPERIMENTAL EM RATOS
}

\author{
Ingestion of prebiotics prevents gastrectomy-induced iron malabsorption and anemia? \\ Experimental study in rats
}

\author{
Elisvânia Freitas dos SANTOS, Kathia Hitomi TSUBOI, Marina Rachel ARAÚJO, Mônica Almeida FALCONI, \\ Arthur C. OUWEHAND, Nelson Adami ANDREOLLO, Celio Kenji MIYASAKA
}

\begin{abstract}
Trabalho realizado no Programa de PósGraduação, Departamento de Alimentos e Nutrição (DEPAN), Faculdade de Engenharia de Alimentos (FEA) e Laboratório de Enzimologia e Carcinogênese Experimental, Faculdade de Ciências Médicas (FCM), Universidade Estadual de Campinas UNICAMP, Campinas. SP, Brasil.
\end{abstract}

DESCRITORES - Prebióticos. Gastrectomia. Ferro. Anemia. Rato.

\section{Correspondência: \\ Elisvânia Freitas dos Santos, \\ e-mail: elisvania@gmail.com \\ Fonte de financiamento: não há \\ Conflito de interesses: não há}

Recebido para publicação: 10/12/2010

Aceito para publicação: 25/01/2011

HEADINGS - Prebiotics. Gastrectomy. Iron. Anemia. Rats.
RESUMO - Racional - Ratos gastrectomizados desenvolvem anemia, e a ingestão de galactooligossacarídeo (GOS) e a polidextrose (PDX) aumenta a absorção de ferro e promove a recuperação da anemia em ratos com deficiência de ferro. Objetivo - Avaliar se GOS e PDX estimulam a absorção de ferro em ratos gastrectomizados e normais. Métodos - Os ratos foram laparotomizadas (controle falso-operado) e parcialmente gastrectomizados (Billroth II) em grupos de 20 animais. Os animais de ambos os tratamentos foram posteriormente submetidos à dieta controle (AIN-93M) ou a mesma dieta suplementada com GOS e PDX ( $25 \mathrm{~g} / \mathrm{kg}$ de dieta cada) durante oito semanas e divididos em quatro subgrupos: sham-operados e gastrectomizados sem GOS e PDX, sham-operados e gastrectomizados com GOS e PDX. Dois animais morreram durante o experimento. Todos os ratos gastrectomizados receberam uma injeção intramuscular de vitamina B-12 a cada duas semanas. Hematócrito $(\mathrm{HCT})$ e concentração de hemoglobina (HGB) foram dosados no início e nos dias 30 e 56 dias após o início da alimentação. No último dia do estudo, o sangue total foi coletado para determinação da concentração de ferro sérico. Resultados - Na dieta com GOS e PDX a excreção de ferro nas fezes foi significativamente menor do que no grupo sem prebióticos. Absorção aparente de ferro e ferro sérico foram maiores nos grupos alimentados com GOS e PDX (ambos grupos: sham operados e gastrectomizados) do que nos grupos não alimentados com GOS e PDX. O HCT e HGB foram significativamente menores nos ratos gastrectomizados que no grupo controle, entretanto, a suplementação com GOS e PDX melhorou os níveis de HGB neste grupo. Conclusão - A associação de GOS e PDX aumenta a absorção de ferro em ratos shamoperados e gastrectomizados e ainda previne a anemia pós-gastrectomia.

ABSTRACT - Background - The ingestion of two prebiotics, galactooligosaccharide (GOS) and polydextrose (PDX), leads to an increase in iron absorption and promotes recovery from anemia in gastrectomized rats. Aim - To study whether GOS + PDX stimulate iron absorption in gastrectomized and normals rats. Methods - Rats were laparotomized (sham-operated control) and partially gastrectomized (Billroth II) in groups of 20 each. Animals from both treatments were subsequently fed a control diet (AIN-93M) or the same diet supplemented with GOS and PDX ( $25 \mathrm{~g} / \mathrm{Kg}$ of diet each) for eight weeks. They were divided into four subgroups, i.e., sham-operated and gastrectomized without GOS + PDX, sham-operated and gastrectomized with GOS + PDX. Two rats died during the experiment. All gastrectomized rats received an intramuscular injection of vitamin B-12 every two weeks. Hematocrit (HCT) and hemoglobin concentration (HGB) were measured at the start and on day 30 and 56 days after the start of feeding. On the final day of the study, total blood was collected for determination of serum iron concentration. Results - In the diet with GOS + PDX, iron excretion in feces was significantly lower than without the prebiotics. Apparent iron absorption and serum iron was higher in the GOS + PDX fed groups (both sham operated and gastrectomized) than in the non-GOS + PDX fed groups. The HCT and $\mathrm{HGB}$ concentrations were significantly lower in gastrectomized rats than in the control group, however, GOS + PDX feeding improved HGB levels in this group. Conclusion The association of the GOS + PDX increase absorption of iron in sham-operated and gastrectomized rats and still prevent postgastrectomy anemia. 


\section{INTRODUÇÃO}

A anemia é complicação comum em pacientes após ressecção gástrica 11,12,24,25. Nas fases iniciais após a gastrectomia, a anemia por deficiência de ferro ocorre porque o ácido gástrico desempenha papel importante na absorção do ferro no intestino delgado através da solubilização de sais de ferro insolúveis ${ }^{14,17,23}$.

Alguns autores têm sugerido que a absorção de ferro parece não ser somente no intestino delgado, o intestino grosso parece contribuir também, mas até que ponto ainda não está totalmente claro ${ }^{12}$. Assim, a absorção de ferro pelo intestino grosso pode contribuir para a recuperação da anemia em ratos deficientes de ferro ${ }^{8,9}$. De fato, tem sido demonstrado que 0 trato digestivo, especialmente 0 de cólon proximal, também pode ser um importante local de absorção de ferro quando a absorção no intestino delgado é insuficiente ${ }^{2,3,8}$ como talvez no caso após a gastrectomia ${ }^{7}$. Além disso, estudo com ratos demonstrou que os ácidos graxos de cadeia curta e, em particular, propionato, parece desempenhar papel no aumento da absorção de ferro no cólon proximal ${ }^{2}$. Essa observação sugere que a ingestão de prebióticos pode aumentar a biodisponibilidade do ferro da dieta. Recentemente, tem havido muitos relatos indicando que os carboidratos indigeríveis estimulam a absorção de minerais ${ }^{1,10,13,18}$.

O objetivo do presente trabalho foi verificar o efeito da suplementação alimentar com uma combinação de dois prebióticos (galactooligossacarídeo e polidextrose) na absorção de ferro em ratos gastrectomizados e normais.

\section{MÉTODOS}

\section{Animais e procedimento cirúrgico}

Quarenta ratos machos Wistar com 250,0 $\pm 5 \mathrm{~g}$ de peso corporal foram mantidos em gaiolas coletivas, em sala com temperatura controlada $\left(22 \pm 1^{\circ} \mathrm{C}\right)$, umidade (60-70\%), 12 horas ciclo dia-noite (luzes acesas às 07:00), com dieta (veja abaixo) e água deionizada ad libitum. Os animais foram divididos aleatoriamente em dois grupos de 20 animais cada. Um grupo foi submetido a vagotomia troncular anterior e gastrectomia parcial (Billroth II). O outro era falso operado a fim de serem submetidos ao mesmo estresse cirúrgico, onde a cavidade abdominal foi mantida aberta por aproximadamente 45 minutos, que é a duração de uma gastrectomia. Os ratos foram anestesiados com tiopental sódico $(25 \mathrm{mg} / \mathrm{Kg}$ de peso corporal/por via intravenosa). O protocolo experimental foi previamente aprovado pelo Comitê de Ética em Experimentação Animal (CEEA) da Universidade Estadual de Campinas - UNICAMP ( ${ }^{\circ}{ }^{\circ} 839-1$ registro, 08/06/2005).

\section{Grupos experimentais e dietas}

Quinze dias após o procedimento cirúrgico, os ratos foram divididos aleatoriamente em quatro subgrupos experimentais: sham vs gastrectomia, que foram divididas em dieta controle vs dieta com galactooligossacarídeo polidextrose. Dois ratos gastrectomizados alimentados com a dieta do grupo controle morreram durante o experimento. Os animais foram alimentados com as dietas experimentais por oito semanas.

As dietas controle e experimentais foram preparadas segundo a formulação AIN-93M, durante oito semanas ${ }^{16}$. Galactooligossacarídeo (GOS, 50-55\% de oligossacarídeos (Yakult SA Indústria e Comércio, São Paulo, SP, Brasil) e polidextrose (PDX, Litesse Ultra, Danisco Brasil Ltda, Cotia, SP, Brasil); cada uma foi adicionada em $25 \mathrm{~g} / \mathrm{kg}$ dieta, substituindo a sacarose da dieta controle.

A Tabela 1 mostra a composição das duas dietas experimentais (controle e GOS + PDX). Metade dos gastrectomizados e metade dos ratos sham-operados foram alimentados com a dieta controle, e os ratos remanescentes em ambos os grupos foram alimentados com a dieta GOS + PDX.

TABELA 1 - Composição das dietas experimentais

\begin{tabular}{|c|c|c|}
\hline Ingredientes, $\mathrm{g} / \mathrm{Kg}$ & Dieta Controle & Dieta com GOS ${ }^{1}+$ PDX $^{2}$ \\
\hline Amido de milho ${ }^{3}$ & 466 & 466 \\
\hline Maltodextrina $^{3}$ & 155 & 155 \\
\hline Caseína $^{4}$ & 140 & 140 \\
\hline Óleo de soja ${ }^{5}$ & 40 & 40 \\
\hline Celulose $^{6}$ & 50 & 50 \\
\hline Sacarose $^{7}$ & 100 & 50 \\
\hline $\operatorname{GOS}^{1}$ & 0 & 25 \\
\hline $\mathrm{PDX}^{2}$ & 0 & 25 \\
\hline Mistura mineral ${ }^{8}$ & 35 & 35 \\
\hline Mistura vitamínica ${ }^{8}$ & 10 & 10 \\
\hline L-Cistina ${ }^{9}$ & 1,8 & 1,8 \\
\hline Bitartarato de colina ${ }^{10}$ & 2,5 & 2,5 \\
\hline Tert-butilhidroquinona ${ }^{11}$ & 0,008 & 0,008 \\
\hline
\end{tabular}

${ }^{1}$ Galactooligossacarídeo (GOS, $50 \mathrm{~g} / \mathrm{Kg}$ de dieta) (50-55\% de oligossacarídeo, fabricado no Japão, Yakult Indústria e Comércio, São Paulo, SP, Brasil)

Polidextrose (PDX, 25 g/Kg diet) Litesse Ultra, Danisco Brasil Ltda, Cotia, SP, Brasil. Polidextrose pó refinado.

${ }^{3}$ Amido de milho e maltodextrina (Corn Products Brasil - Ingredientes Indústria Ltda, Mogi Guaçu, SP, Brasil).

${ }^{4}$ Plury Química Ltda, Diadema, SP, fabricado por Naarden Agro Products-Holland.

${ }^{5}$ Marca Liza, Cargill do Brasil, Uberlândia, MG, Brasil.

${ }^{6}$ Marca Microcel, Blanver Farmoquímica Ltda, Cotia, SP, Brasil.

${ }^{7}$ Refinaria União, Assis, SP, Brasil.

${ }^{8}$ Preparada de acordo com a formulação AIN-93M 16. Formulada por M. Cassab Comércio e Indústria Ltda, São Paulo, SP, Brasil.

${ }_{9}^{9}$ Marca Synth C1027.01.AE; Diadema, SP, Brasil.

10, 11 SIGMA Chemical Co., St. Louis, Mo, USA.

Os animais tinham livre acesso à água deionizada durante todo o período experimental. Para a prevenção da anemia megaloblástica, os ratos gastrectomizados receberam suplementos de vitamina B12 (Cianocobalamina 0,5 mg/kg/intramuscular Cianotrat 5000 - Instituto Terapêutico Delta Ltda, Indaiatuba, SP, Brasil) a cada duas semanas, começando 
uma semana depois da operação. Os animais do grupo sham receberam cloreto de sódio a 0,9\% (Sanobiol Ltda, Pouso Alegre, MG, Brasil).

Para a coleta de fezes, os animais foram colocados em gaiolas metabólicas individuais, durante três dias nos períodos de 15,35 e 55 do experimento ${ }^{5}$.

O ganho de peso e o consumo da dieta dos animais foram monitorados três vezes por semana, durante oito semanas.

\section{Métodos analíticos}

Sangue de animais anestesiados foi coletado por punção da veia retro-ocular a cada quatro semanas durante o período experimental. No início do experimento, o sangue foi coletado de animais aleatoriamente antes do procedimento cirúrgico. Amostras de sangue foram analisadas para determinar o hematócrito e hemoglobina, usando o analisador hematológico (Advia TM 120, Bayer ${ }^{\circledR}$, Irlanda).

A concentração de ferro sérico foi determinada por método colorimétrico comercialmente disponível (Laborlab, Guarulhos,SP, Brasil).

As fezes obtidas foram secas, pesadas e moídas. As dietas e as fezes em pó secas foram calcinadas em temperaturas aumentadas de forma linear até $550^{\circ} \mathrm{C}$ por $6 \mathrm{~h}$, e depois a $550^{\circ} \mathrm{C}$ por $18 \mathrm{~h}$ na mufla (Indústria Fornitec e Comércio Ltda, São Paulo, SP, Brasil). As amostras foram digeridas com $0,5 \mathrm{ml}$ concentrado de $\mathrm{HNO}_{3}(65 \%)$ e $0,15 \mathrm{ml}(30 \%) \mathrm{H}_{2} \mathrm{O}_{2}$ (Merck do Brasil, São Paulo, SP, Brasil), em tubos fechados sob pressão Hostaflon aquecidas no micro-ondas (DGT-100 Plus Provecto, Jundiaí, SP, Brasil). As determinações de ferro nas fezes e na dieta foram realizadas por emissão óptica IRIS-AP (Thermo Jarrell Ash, Franklin - Massachusetts EUA) no laboratório especializado Biominerais Análises Químicas, Campinas, SP, Brasil e os cálculos realizados foram: absorção aparente de ferro $(\mathrm{mg} / \mathrm{dia})=$ consumo de ferro (mg/dia) - excreção de ferro nas fezes (mg/ dia) $)^{5}$.

\section{Análise estatística}

Os resultados foram submetidos à análise de variância (ANOVA), com o uso do teste de Duncan para a comparação das médias. Os dados foram analisados por duas vias (tratamento e dieta) ou três vias (tratamento, dieta e tempo). As diferenças foram consideradas significativas quando $p<0,05$. Os dados foram expressos como média e erro-padrão da média (EPM) (Statistica 6.0 ${ }^{\circledR}$, Statsoft, Inc. Tulsa, USA,) para Windows ${ }^{25}$.

\section{RESULTADOS}

Ganho de peso e ingestão alimentar (Tabela 2)

Peso corporal inicial em ambos os grupos shamoperados foi significativamente maior do que nos ratos gastrectomizados correspondentes $(p<0,05)$. Também no final do estudo, o peso corporal final em ambos os grupos sham-operados foi significativamente maior do que os ratos gastrectomizados $(p<0,05)$. No entanto, o ganho de peso corporal total não foi significativamente diferente entre os grupos sham e gastrectomizados $(p>0,05)$. O consumo alimentar em ratos gastrectomizados foi significativamente menor que nos ratos sham-operados, independentemente da dieta $(p<0,05)$.

TABELA 2 - Peso corporal inicial e final e consumo alimentar de ratos sham-operados e gastrectomizados alimentados com dietas com GOS + PDX $(\mathrm{G}+\mathrm{P})$ ou sem GOS + PDX (G+P) (controle)

\begin{tabular}{ll|l|c|c|}
\hline Tratamento / Dietas & Peso inicial (g) & Peso final (g) & $\begin{array}{c}\text { Ganho de peso } \\
\text { total (g/8 wk) }\end{array}$ & $\begin{array}{c}\text { Ingestão alimentar } \\
\text { (g/dia) }\end{array}$ \\
\hline Sham & & & & \\
\hline $\begin{array}{l}\text { Controle }(n=10) \\
\text { G+P }(n=10)\end{array}$ & $264,50 \pm 3,43 a$ & $421,60 \pm 7,66 a$ & $157,10 \pm 8,99 a$ & $25,84 \pm 2,48 a$ \\
\hline Gastrectomia & $255,20 \pm 8,28 a$ & $399,00 \pm 8,33 a$ & $143,80 \pm 7,63 a$ & $27,09 \pm 3,98 a$ \\
\hline Controle $(n=10)$ & $221,88 \pm 4,74 b$ & $375,00 \pm 9,94 b$ & $153,13 \pm 6,62 a$ & $21,02 \pm 1,10 b$ \\
\hline$G+P(n=10)$ & $219,30 \pm 6,73 b$ & $381,70 \pm 9,60 b$ & $162,40 \pm 9,05 a$ & $22,14 \pm 1,81 b$ \\
\hline
\end{tabular}

Cada valor representa à média \pm erro-padrão da média (EPM). Valores em uma coluna que não compartilham a mesma letra são significativamente diferente, $\mathrm{p}<0,05$ (Duncan's test). Para mais detalhes sobre a dieta, consulte a Tabela 1

\section{Peso úmido e seco das fezes (Tabela 3)}

Em ratos sham-operados e gastrectomizados, a média de peso seco e úmido das fezes foi significativamente maior nos ratos que receberam dieta com GOS + PDX do que nos que receberam dieta controle (sem GOS + PDX) $(p<0,05)$. No entanto, não houve diferença entre os ratos sham-operados e gastrectomizados para a mesma dieta $(p>0,05)$.

TABELA 3 - Peso úmido e seco das fezes (g), coletados em três vezes (15, 35 e 55 dias) da fase experimental, durante três dias em cada período dos ratos sham-operados e gastrectomizados alimentados com dietas com GOS + PDX ou sem GOS + PDX (controle)

\begin{tabular}{lc|c}
\hline \multirow{2}{*}{ Grupos / Tratamentos } & \multicolumn{2}{c}{ Peso da fezes $(\mathrm{g})$} \\
\cline { 2 - 3 } & Peso umido $(\mathrm{g})$ & Peso seco $(\mathbf{g})$ \\
\hline Sham & & \\
Controle $(n=10)$ & $4,62 \pm 0,22 b$ & $4,12 \pm 0,17 b$ \\
G+P $(n=10)$ & $6,95 \pm 0,25 a$ & $5,99 \pm 0,20 a$ \\
\hline Gastrectomia & & \\
Controle $(n=10)$ & $5,42 \pm 0,46 b$ & $4,73 \pm 0,35 b$ \\
\hline$G+P(n=10)$ & $6,83 \pm 0,25 a$ & $5,89 \pm 0,19 a$ \\
\hline
\end{tabular}

Cada valor representa à média \pm erro-padrão da média (EPM). Valores em uma coluna que não compartilham a mesma letra são significativamente diferente, $\mathrm{p}<0,05$ (Duncan's test). Para mais detalhes sobre a dieta, consulte a Tabela 1

\section{Concentração de hematócrito e hemoglobina (Figuras 1 e 2) \\ Os níveis de hematócrito iniciais foram} semelhantes em ambos os animais sham-operados e gastrectomizados (Figura 1). Após a quarta e oitava 
semanas do experimento, os níveis de hematócrito dos ratos sham-operados permaneceram a um nível semelhante como no início do experimento e foram superiores aos níveis de hematócrito dos animais gastrectomizados $(p<0,05)$.
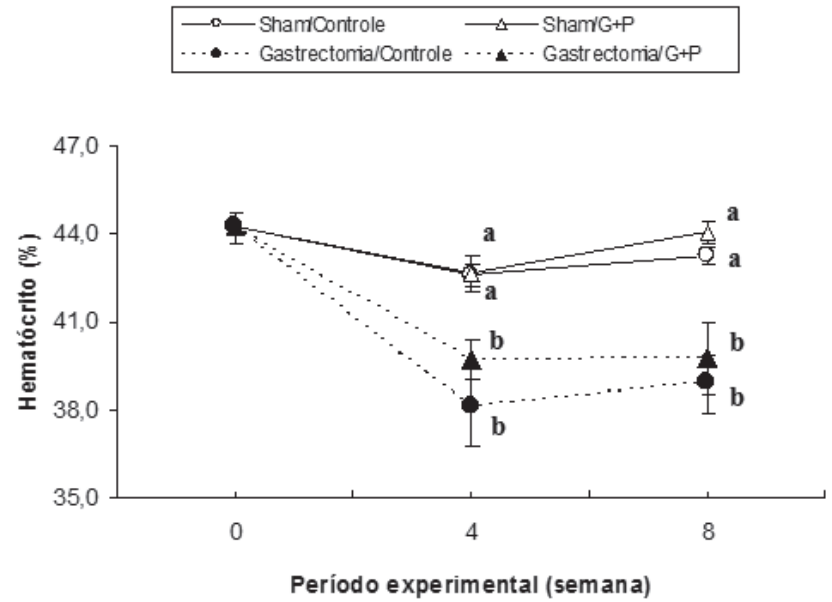

FIGURA 1 - Concentração de hematócrito em ratos sham-operados ou gastrectomizados alimentados com dietas contendo (GOS + POL) ou sem GOS + PDX (controle) em 0, 4 e 8 semanas após o início do período experimental. Cada valor representa a média \pm EPM (Sham/Controle: $\mathrm{n}=10 ;$ Sham/G + P: $\mathrm{n}=10$; Gastrectomia/Controle: $\mathrm{n}=8$; Gastrectomia/G + P: n=10). Dieta (controle ou GOS + PDX), tratamento (com ou sem a gastrectomia) e tempo $(0,4$ e 8 semanas). Os pontos que não partilham a mesma letra são significativamente diferente, $p<0,05$ (Duncan's test). Para mais detalhes sobre a dieta, consulte a Tabela 1

Os níveis de hemoglobina iniciais foram semelhante para os ratos gastrectomizados e sham-operados (Figura 2). Na quarta e oitava semana do experimento, a hemoglobina foi maior nos ratos sham-operado com dieta controle e dieta com GOS + PDX quando comparados aos animais gastrectomizados $(p<0,05)$. No entanto, os níveis de hemoglobina em ratos gastrectomizados recebendo dieta GOS + PDX foram significativamente maiores do que em ratos gastrectomizados que receberam dieta controle $(p<0,05)$, apesar de não chegarem ao mesmo nível que os ratos sham-operado.

\section{Concentração de ferro sérico (Figura 3)}

Em ratos sham-operado, após oito semanas, o ferro sérico foi significativamente maior nos ratos que receberam dieta com GOS + PDX do que nos que receberam dieta controle (sem GOS + PDX) $(p<0,05)$.

$\mathrm{Em}$ ratos gastrectomizados o ferro sérico foi significativamente menor após oito semanas em relação ao sham-operado, independentemente da dieta $(p>0,05)$. Contudo, os ratos que receberam a suplementação de GOS + PDX tiveram concentrações de ferro sérico significativamente mais elevadas, do que os que receberam a dieta controle (sem GOS + PDX) $(p<0,05)$.

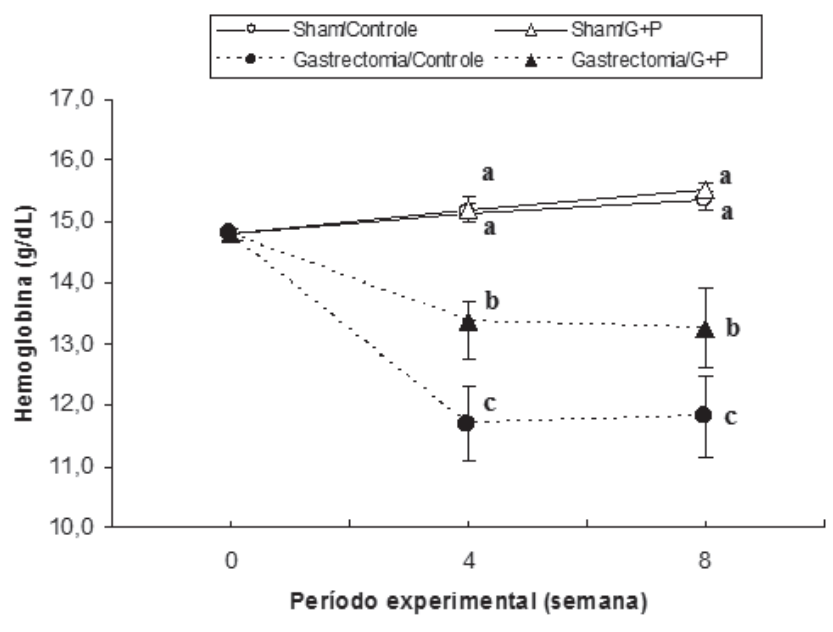

FIGURA 2 - Concentração de hemoglobina em ratos sham-operados ou gastrectomizados alimentados com dietas contendo (GOS + POL) ou sem GOS + PDX (controle), em 0, 4 e 8 semanas após o início do período experimental. Cada valor representa a média \pm EPM (Sham/Controle: $\mathrm{n}=10$; Sham/G + P: n=10; Gastrectomia/Controle: $\mathrm{n}=8$; Gastrectomia/G + P: n=10). Dieta (controle ou GOS + $\mathrm{PDX}$ ), tratamento (com ou sem a gastrectomia) e tempo $(0,4$ e 8 semanas). Os pontos que não partilham a mesma letra são significativamente diferente, $p<0,05$ (Duncan's test). Para mais detalhes sobre a dieta, consulte a Tabela 1

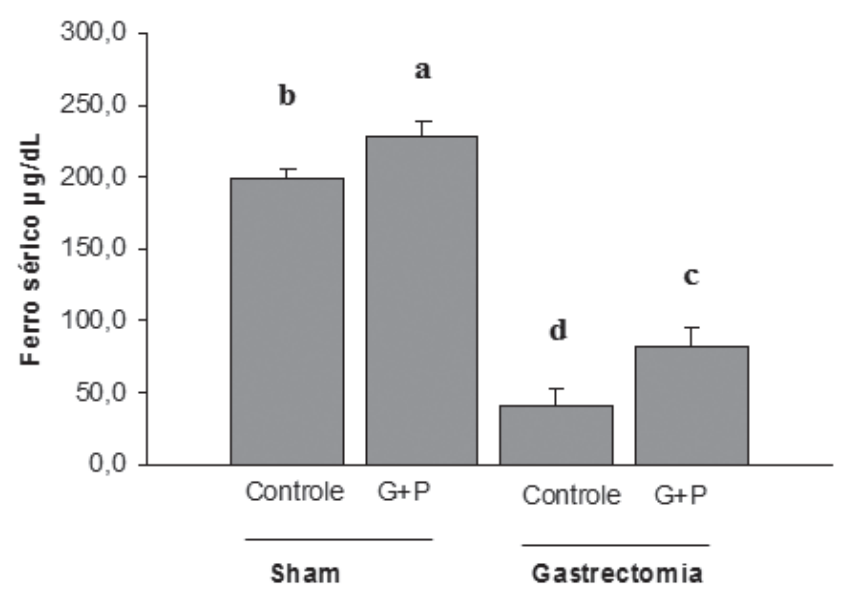

FIGURA 3 - Ferro sérico em ratos sham-operados ou gastrectomizados alimentados com dietas contendo (GOS + PDX) ou sem GOS + PDX (controle). Cada valor representa a média \pm EPM (Sham/Controle: $n=10 ;$ Sham $/ G+P$ : $\mathrm{n}=10$; Gastrectomia/Controle: $\mathrm{n}=8$; Gastrectomia/G + P: $n=10$ ). Barras que não compartilham a mesma letra são significativamente diferentes, $\mathrm{p}<0,05$ (Duncan's test). Para mais detalhes sobre a dieta, consulte a Tabela 1

Ferro fecal e absorção aparente de ferro (Figuras 4 e 5 )

A concentração de ferro nas fezes dos ratos shamoperados e gastrectomizados que receberam GOS + PDX na dieta foi menor $(p<0,05)$ em comparação à dieta controle, mas não foi diferente entre os ratos sham-operados e gastrectomizados (Figura 4). 


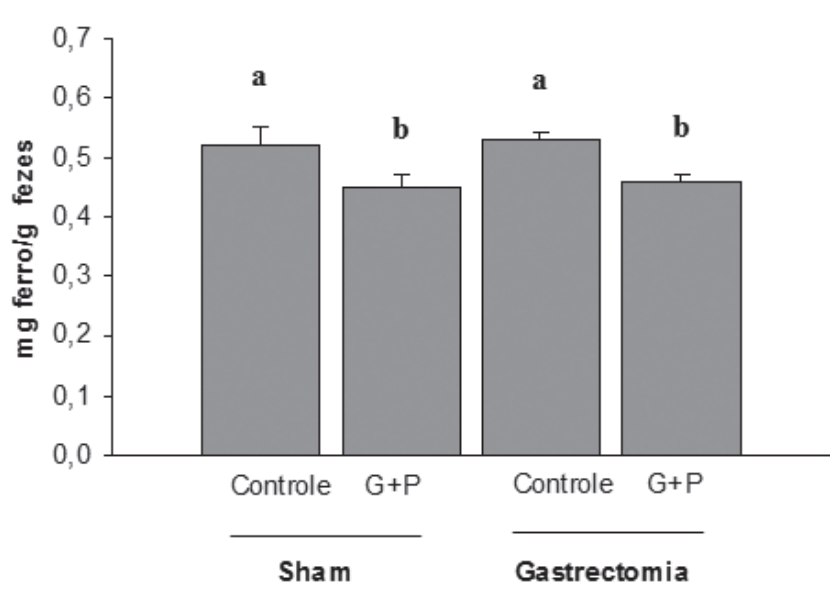

FGURA 4 - Concentração de ferro nas fezes (mg/g de fezes) em ratos sham-operados ou gastrectomizados alimentados com dietas contendo (GOS + PDX) ou sem GOS + PDX (controle). Cada valor representa a média \pm EPM (Sham/ Controle: $n=10$; Sham/G + P: n=10; Gastrectomia/ Controle: $n=8$; Gastrectomia/G + P: $n=10$ ). Barras que não compartilham a mesma letra são significativamente diferentes, $p<0,05$ (Duncan's test). Para mais detalhes sobre a dieta, consulte a Tabela 1

A absorção aparente de ferro em sham-operados foi significativamente maior $(p<0,05)$ no grupo que recebeu o GOS + PDX na dieta, em comparação com os ratos que receberam a dieta controle (Figura 5). A absorção aparente de ferro de ratos gastrectomizados foi significativamente menor em comparação com os shamoperados recebendo a mesma dieta $(p<0,05)$. No entanto, os que receberam o GOS + PDX na dieta, apresentaram absorção aparente de ferro significativamente maior

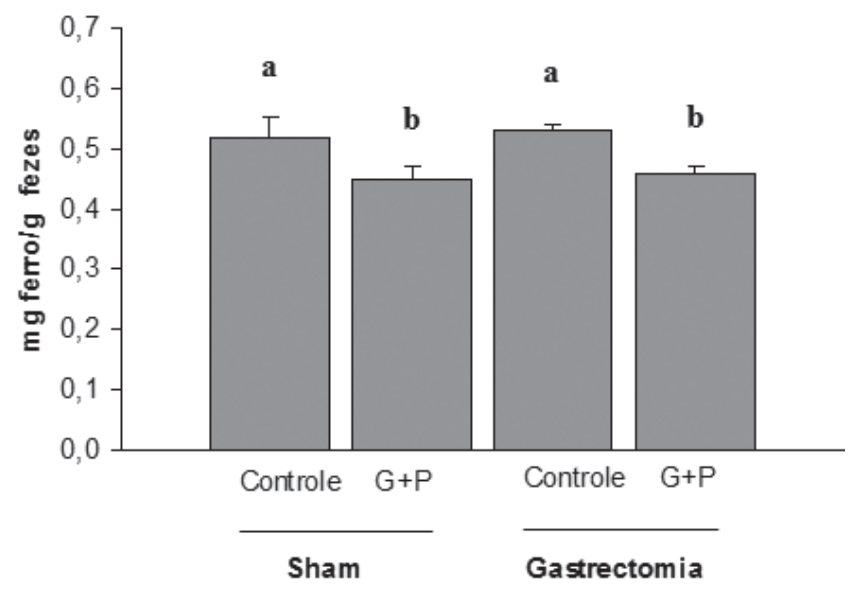

FIGURA 5 - Absorção aparente de ferro em ratos sham-operados ou gastrectomizados alimentados com dietas contendo (GOS + PDX) ou sem GOS + PDX (controle). Cada valor representa a média \pm EPM (Sham/Controle: $\mathrm{n}=10 ;$ Sham $/ \mathrm{G}+\mathrm{P}: \mathrm{n}=10 ;$ Gastrectomia/Controle: $\mathrm{n}=8$; Gastrectomia/G + P: $\mathrm{n}=10$ ). Barras que não compartilham a mesma letra são significativamente diferentes, $p<0,05$ (Duncan's test). Para mais detalhes sobre a dieta, consulte a Tabela 1 do que nos alimentados com dieta controle do mesmo grupo $(p<0,05)$ e foi elevado a um nível semelhante ao dos ratos sham-operados com dieta controle.

\section{DISCUSSÃO}

Como observado anteriormente ${ }^{23}$, a absorção de ferro foi severamente diminuída pelo gastrectomia parcial (Figura 5). Estudos anteriores mostraram que a gastrectomia parcial e vagotomia troncular anterior foram um bom modelo experimental para estudar o metabolismo do ferro ${ }^{21}$ e metabolismo do cálcio ${ }^{22}$. No entanto, a etiologia da má absorção de ferro após gastrectomia não foi totalmente esclarecida. Mucina gástrica tem sido relatada a ser envolvida na via integrina-mobilferrina, que é um dos caminhos proposto de transporte de ferro no intestino delgado, sugerindo que a falta de mucina gástrica é responsável pela má absorção de ferro induzida pela gastrectomia ${ }^{23}$. A má absorção de ferro (Figura 5) leva à anemia, como indicado pela redução da hemoglobina, hematócrito e os níveis séricos de ferro (Figuras 1, 2 e 3 ).

A deficiência de ferro é um problema mundial de saúde: ela prejudica o crescimento, desenvolvimento cognitivo e imunidade. A baixa taxa de absorção de ferro na dieta é principalmente devido a sua baixa solubilidade em $\mathrm{pH}$ alcalino e ao pequeno segmento do intestino proximal disponíveis para a absorção de ferro específicos. Os efeitos positivos da fermentação bacteriana na absorção de minerais, como o ferro, sugere que o cólon pode contribuir significativamente para a sua disponibilidade. Mecanismos de absorção de ferro pelo cólon não são bem compreendidos e os ácidos graxos de cadeia curta parecem desempenhar um papel importante 2 .

No colo proximal de ratos com deficiência de ferro, a absorção de seis minerais foi maior que no duodeno, jejuno e íleo e também foi maior que nos ratos do grupo controle (dieta com ferro). Para todos os minerais, o aumento foi o resultado da absorção passiva aumentada. Sugerindo que a anemia ferropriva aumenta a permeabilidade da membrana do cólon. Os resultados apóiam a hipótese de que em estados diferentes, incluindo a anemia ferropriva em que a capacidade do intestino de absorver nutrientes fica comprometida, a mucosa do cólon aparentemente compensa a funcionalidade prejudicada do intestino delgado ${ }^{3}$, como após a gastrectomia (Billroth II).

Além disso, estudos em animais revelaram que os ácidos graxos de cadeia curta, em especial propionato parece aumentar a absorção de ferro no cólon proximal ${ }^{2}$. Isso pode indicar um potencial mecanismo pelo qual a ingestão de prebióticos aumentam a biodisponibilidade do ferro dietético. Sakai, et al. ${ }^{18}$ mostraram que a fermentação cecal de fruto-oligossacarídeos está envolvido na prevenção da anemia induzida por ressecção gástrica. 
Estudos nossos anteriores mostraram que a administração de polidextrose aumentam a absorção do ferro ${ }^{19}$ e cálcio ${ }^{20}$ em ratos gastrectomizados. Um estudo in vitro de modelagem de fermentação colônica, mostraram que a administração polidextrose aumenta o nível de bifidobactérias e os níveis de ácidos orgânicos de cadeia curta, ácido acético em particular. Além disso, a polidextrose reduziu os níveis de bactérias potencialmente patogênicas no cólon proximal ${ }^{15}$. Consequentemente, especula-se que neste estudo o efeito da polidextrose em aumentar a absorção de ferro em ratos gastrectomizados aconteceu no intestino grosso. Além disso, alimentação com GOS aumentou a absorção de minerais ${ }^{4,5,6}$. Chonan e Watanuki ${ }^{4,5,6}$ demonstraram os efeitos estimulantes do GOS na absorção de cálcio pode ser parcialmente associado a solubilidade aumentada do cálcio e do conteúdo fluido no lúmen intestinal.

No presente estudo, a gastrectomia parcial causou anemia devido absorção de ferro reduzido; este fato poderia ser parcialmente evitado através da inclusão de GOS + PDX na dieta (Figuras 2, 3 e 5). Ratos gastrectomizados receberam vitamina B12 por via subcutânea, para evitar a anemia perniciosa. Os resultados aqui apresentados indicam que a anemia pós-gastrectomia observada neste estudo foi de anemia ferropriva. Estes resultados podem ser benéficos para pacientes com anemia pós-gastrectomia. Entretanto, evidências adicionais são necessárias para se adaptar ao tratamento clínico dos seres humanos.

\section{CONCLUSÃO}

A suplementação com GOS + PDX preveniu má absorção de ferro e anemia em ratos pós-gastrectomia, e o intestino grosso parece estar envolvido no processo de absorção de ferro em ratos gastrectomizados.

\section{AGRADECIMENTOS}

Ao Conselho Nacional de Desenvolvimento Científico e Tecnológico (CNPq), Laboratório Biominerais Análises Químicas Ltda e Johnson \& Johnson ${ }^{\circledR}$, pelos fios cirúrgicos.

\section{REFERÊNCIAS}

1. Afsana $\mathrm{K}$, Shiga $\mathrm{K}$, Ishizuka $\mathrm{S}$, Hara H. Ingestion of an indigestible saccharide, difructose anhydride III, partially prevents the tannic acid-induced suppression of iron absorption in rats. J. Nutr. 2003; 133: 3553-60.

2. Bouglé $D$, Vaghefi-Vaezzadeh $N$, Roland $N$, Roland $N$, Bouvard G, Arhan P, Bureau F, Neuville D, Maubois JL. Influence of shortchain fatty acids on iron absorption by proximal colon. Scand. J. Gastroenterol. 2002; 37(9): 1008-1011.

3. Campos MS, Gomez-Ayala AE, Lopez-Aliaga I, Pallares I, Hartiti S, Pharm B, Alferez MJ M, Barronuevo M. Role of the proximal colon in mineral absorption in rats with and without ferropenic anemia. Nutr. Res. 1996; 16(9): 1529-1543.
4.Chonan O, MatsumotoK, WatanukiM.Effectofgalactooligosaccharides on calcium absorption and preventing bone loss in ovariectomized rats. Biosci. Biotech. Biochem. 1995;59(2):236-239.

5. Chonan O, Watanuki M. Effect of galactooligasaccharides on calcium absorption in rats. J. Nutr. Sci. Vitaminol. 1995;41(1): 95-104.

6. Chonan O, Watanuki M. The effect of 6 '-Galactooligosaccharides on bone mineralization of rats adapted to different leves of dietary calcium. Int J Vitam. Nutr Res. 1996; 66(3): 244-49.

7. Diniz MFHS, Diniz MTC. Obesidade mórbida. In: Fonseca JGM. Obesidade e outros distúrbios alimentares. Rio de Janeiro: Médica e Científica. Parte C, v.2, 2001, p.290-293.

8. Ebihara K, Okano J, Miyata T. Comparison of ferrous and ferric iron bioavailability following rat cecal infusion. Nutr. Res. 1994;14(2): 221-228.

9. Ebihara K, Okano J. Comparison of bioavailability and hemoglobin repletion of ferric and ferrous iron infused into the cecum in anemic rats. Nutr. Res. 1995;15: 889-897.

10. Hara $H$, Suzuki T, Aoyama Y. Ingestión of the soluble dietary fibre, polydextrose, increases calcium absorption and bone mineralization in normal and total-gastrectomized rats. Br. J. Nutr. 2000; 84: 655-661.

11. Ohta A, Ohtsuki M, Hosono A, Adachi T, Hara H, Sakata T. Dietary fructooligosaccharides prevent osteopenia after gastrectomy in rats. J Nut. 1998;128(1): 106-110.

12. Ohta A, Ohtsuki M, Uehara M, Hosono A, Hirayama M, Adachi T, Hara $\mathrm{H}$. Dietary fructooligosaccharides prevent postgastrectomy anemia and osteopenia in rats. J Nut. 1998; 128(3): 485-90.

13. Ohta A, Sakai K, Takasaki M, Uehara M, Tokunaga T, Adachi T. Dietary heme iron does not prevent postgastrectomy anemia but fructooligosaccharides improve bioavailability of heme iron in rats. Int. J. Vitam. Nutr. Res. 1999; 69(5): 348-355.

14. Papini-Berto SJ, Burini RC. Causas da desnutrição pósgastrectomia. Arq Gastroenterol. 2001;38(4): 272-75.

15. Probert HM, Apajalahti JHA, Rautonen N, Stowell J, Gibson GR. Polydextrose, lactitol, and fructo-oligosaccharide fermentation by colonic bacteria in a three-stage continuous culture system. Appl. Environ. Microbiol. 2004;70(8): 4505-4511.

16. Reeves PG, Nielson FH, Fahey GCJR. AIN-93 purified diets for laboratory rodents: final report of the American Institute of Nutrition ad hoc writing committee on the reformulation of the AIN-76A rodent diet. J Nut. 1993;123(11): 1939-51.

17. Roviello F, Fotia G, Marrelli D, Stefano A, Macchiarelli R, Pinto E. Iron deficiency anemia after subtotal gastrectomy for gastric cancer. Hepatogastroenterology. 2004;51(59): 1510-1514.

18. Sakai K, Ohta A, Shiga K, Takasaki M, Tokunaga T, Hara H. The cecum and dietary short-chain fructooligosaccharides are involved in preventing postgastrectomy anemia in rats. J Nut. 2000; 130(6): 1608-12.

19. Santos EF, Tsuboi $\mathrm{KH}$, Araújo MR, Falconi MA, Ouwehand $A C$, Andreollo NA, Miyasaka CK. Ingestion of polydextrose increase the iron absorption in rats submitted to partial gastrectomy. Acta Cir Bras. 2010:25(6)518-524.

20. Santos EF, Tsuboi KH, Araújo MR, Ouwehand AC, Andreollo NA, Miyasaka CK. Dietary polydextrose increase calcium absorption in normal rats. ABCD Arq Bras Cir Dig 2009;22(4):201-5.

21. Santos EF, Tsuboi KH, Palu BF, Araújo MR, Andreollo NA, Miyasaka CK. Effects of the partial gastrectomy associated to anterior truncal vagotomy in the metabolism of the iron in rats. Acta Cir Bras. 2008;23(5):401-4.

22. Santos EF, Tsuboi KH, Palu BF, Araújo MR, Andreollo NA, Miyasaka CK. Partial gastrectomy associated to anterior truncal vagotomy: alterations in metabolism of calcium. Experimental study in rats. Arq Bras Cir Dig. 2009;22(2)105-9.

23. Shiga K, Nishimukai M, Tomita F, Hara H. Ingestion of difructose anhydride III, a non-digestible disaccharide, prevents gastrectomyinduced iron malabsorption and anemia in rats. Nutrition. 2006; 22(7-8): 786-93.

24. Statsoft, Inc. Statistica for Windows [Computer program manual]. Tulsa, USA, (2000) Available from http://www.statsoft.com

25. Tovey FI, Hobsley M. Post-gastrectomy patients need to be followed up for 20-30 years. World J Gastroenterol. 2000; 6:45-8. 